\title{
Negative contrast as a function of reinforcement location and consistent vs. varied reward magnitude
}

\author{
RICHARD S. CALEF, RUTH A. CALEF, and ANDREW D. PROCHASKA \\ West Virginia Wesleyan College, Buckhannon, West Virginia 26201
}

and

\author{
E. SCOTT GELLER \\ Virginia Polytechnic Institute and State University, Blacksburg, Virginia 24061
}

\begin{abstract}
Subsequent to receiving small reward magnitude in the straight runway during Stage 1, rats were given consistently large magnitude of reward or varied large and small reward during placement trials (Stage 2) in a housing cage or in the goalbox. During Stage 3, only animals receiving consistently large magnitude of reward in goalbox placement trials showed a successive negative contrast effect. The results were supportive of frustration theory.
\end{abstract}

Research in instrumental conditioning is replete with data showing the effect of shift in reward magnitude (Black, 1968). Specifically, many studies have shown that rats shifted from large (L) to small (S) magnitude of reward run more slowly than rats maintained on small magnitude of reward, a phenomenon designated as the negative contrast effect (NCE) (e.g., Capaldi \& Lynch, 1967; Crespi, 1942; Ehrenfreund, 1971; Gonzales, Gleitman, \& Bitterman, 1962). It should be noted that in all previous runway studies showing a NCE, large reward magnitude was contingent on a traversal down the alley. One purpose of the present study was to examine whether the NCE could be obtained when large reward magnitude was not contingent on traversing the alley in a multiple-shift paradigm (SLS), but instead presented during placement trials. If a NCE is obtained with such a paradigm, theories previously successful in explaining the NCE would now have to account for the phenomenon occurring even though large reward is received noncontingent on a traversal down the alley.

Theoretically, depressed postshift performance following a shift from large to small magnitude of reward is expected by several interpretations of rewardshift effects (e.g., Amsel, 1958; Capaldi, 1967). Amsel's conditioned frustration theory predicts that subjects shifted from large to small magnitude of reward should run more slowly during postshift training than a control group maintained on small reward. This is regarded as a consequence of conditioned interfering responses elicited by frustration stimuli, which, in turn, result from the downward shift in incentive.

Capaldi's (1967) stimulus aftereffect theory can

Reprint requests should be sent to E. Scott Geller, Virginia Polytechnic Institute and State University, Blacksburg, Virginia 24061. also explain the NCE. According to Capaldi, the reward magnitude received on any trial provides magnitudespecific stimuli present when the organism responds on the next trial. Rats shifted from large to small reward magnitude should run more slowly than controls because the novel aftereffect of the new reward for experimental subjects has not had as much habit strength accrued to it as has the small-reward aftereffect present for controls. However, there has been evidence that the NCE is a frustration phenomenon rather than a result of a stimulus-generalization decrement (Calef, 1972; Calef, Hopkins, McHewitt, \& Maxwell, 1973; Maxwell, Calef, Murray, Shepard, \& Norville, 1976). The present study attempted to provide additional evidence that Amsel's (1958) frustration theory is the best incentive-shift formulation.

Subsequent to receiving small reward magnitude in the straight runway during Stage 1 of the present study, experimental rats were given large magnitude of reward during placement trials in a housing cage or in the goalbox (i.e., Stage 2). During Stage 3, all animals received small reward following a runway response. A control group received small reward throughout the study. Amsel's (1958) frustration theory would predict a NCE during Stage 3 if the placements were given in the goalbox only, since the cues of the goalbox are similar to those of the alley (greater rg-sg, frustration effect, and rf-sf). In other words, frustration theory would predict a NCE even though large reward is received noncontingently during a placement trial. According to Amsel, the only prerequisite to a NCE should be "expectancy" (rg-sg) of large reward while the subject receives small reward. This expectancy should be classically conditioned to the goalbox, hence a running response should not be necessary for the development of this anticipatory construct. However, in 
order for a NCE to occur in Stage 3, the "expectancies" for large reward should be established in the presence of stimuli (goalbox cues) similar to that of the black runway. This could occur only if placement trials are received in the black goalbox and not in a white housing cage. It is noteworthy that Amsel and Hancock (1957) showed a greater "frustration effect" (increase in response vigor following nonreward of a response that previously was rewarded) for those animals receiving a brightness-correlated rather than an uncorrelated goalbox and runway section. The present study also manipulated the type of reward (varied or consistent) received during placement trials.

\section{METHOD}

\section{Subjects}

The subjects were 60 experimentally naive, male albino rats of the Sprague-Dawley strain, approximately 80 days old at the beginning of the experiment. The animals were acquired from Flow Laboratories, Dublin, Virginia, where they had been bred and raised.

\section{Apparatus}

The runway apparatus was essentially the same as that used by Ludvigson and Gay (1966), except that only one of the multiple parallel alleys was used. The alley was comprised of a $33.02-\mathrm{cm}$ gray startbox, a $66.04-\mathrm{cm}$ black run section, and a $30.48-\mathrm{cm}$ black goalbox. The inner width and height of each section of the runway was $7.62 \mathrm{~cm}$. Photocell and clock circuitry provided independent measures of traversal times over the first 20.32-cm (start time) and second 30.48-cm (run-time) segments of the run section. The apparatus contained a solenoid operate, opaque, guillotine-type retract door separating the goal from the alley section, and an opaque Plexiglas ceiling. An exhaust fan mounted in the laboratory provided ventilation and served to mask external noises.
\end{abstract}

\section{Procedure}

Fourteen days prior to the first day of training (Day 15), all rats were placed on a 23-h food-deprivation schedule, which was maintained throughout the study. On Days $7-14$, the rats were taken from their individual home cages and handled for about 2 min each. On Days 13 and 14, the rats were placed in individual carrying cages and taken to the experimental laboratory, where they were allowed to explore start and run sections of the apparatus, while clocks, photocells, and the doors were operated. On those days, approximately $1 \mathrm{~g}$ of $45-\mathrm{mg}$ Noyes pellets, identical to the subsequent reinforcement pellets, was incorporated into the rats' daily food ration.

Ten rats were randomly assigned to each of the six groups. During Phase 1, all six groups received 30 trials of small reward (one pellet) in a straight black runway. During Phase 2 , in which all six groups received 42 placement trials, Group $\mathrm{SL}_{\mathrm{hc}} \mathrm{S}$ received large reward ( 12 pellets) in a white plastic housing cage (all the rats in the present study were housed in wire-mesh cages), while Group $\mathrm{SL}_{\mathrm{gb}} \mathrm{S}$ received large reward in the black goalbox. Group $\mathrm{SL} / \mathrm{S}_{\mathrm{nc}} \mathrm{S}$ received varied large and small reward alternately in the housing cage, while Group $S L / S_{\text {gb }} S$ received varied large and small reward alternately in the goalbox. The two remaining control groups, $\mathrm{SS}_{\mathrm{hc}} \mathrm{S}$ and $\mathrm{SS}_{\mathrm{gb}} \mathrm{S}$, received small reward throughout the second phase, in the home cage or in the goalbox, respectively. During Phase 3, the consistent smallreward conditions of Phase 1 were reinstated.

All groups received one trial on the first day of training, two trials on the second day, and three trials on every day thereafter. Trials were administered to three squads of 10 rats each, consisting of all animals from each group. The running order of subjects within a squad was randomized from day to day. The intertrial interval was approximately $30 \mathrm{~min}$, since a subject did not receive its second daily trial until all subjects in the squad had completed the first trial. The running trials during Phases 1 and 3 were initiated by placing the rat in the startbox. After a 3-sec orientation period, the start door was opened and the rat was allowed to traverse the runway. Following the rat's entry into the goalbox, the retrace door was dropped. The rat was allowed to consume the pellet and was then removed from the apparatus to a nearby holding cage to await its next trial.

\section{RESULTS}

Start and run times were converted to reciprocals, yielding start and run speeds, respectively. The startand run-speed data were essentially similar, but differences were generally larger and more reliable in the run measure. Therefore, only run speeds are presented below.

\section{Phase 1: Preshift Training}

During the final 3 days of Phase 1, only minimum between-groups variation was observed. A simple analysis of variance yielded no significance $(p<.05)$.

\section{Phase 3: Postshift (NCE)}

Figure 1 illustrates group mean speeds as a function of days for subjects receiving their placement trials in the goalbox. As can be seen in Figure 1, only the group receiving consistently large reward in the goalbox (Group SLS) during Phase 2 demonstrated slower running speeds than that shown by small-reward controls (Group SSS) on Day 28 (NCE).

Group mean speeds as a function of days for rats receiving their placement trials in the home cage are presented in Figure 2. As can be seen in Figure 2, a reliable NCE was not observed, since neither Group SLS nor Group SL/SS produced speeds significantly slower than Group SSS.

A treatment (large, small, and varied reward during placement trials) by location (home cage vs. goalbox) analysis of variance was performed on the data from Day 28. The analysis yielded a significant effect of

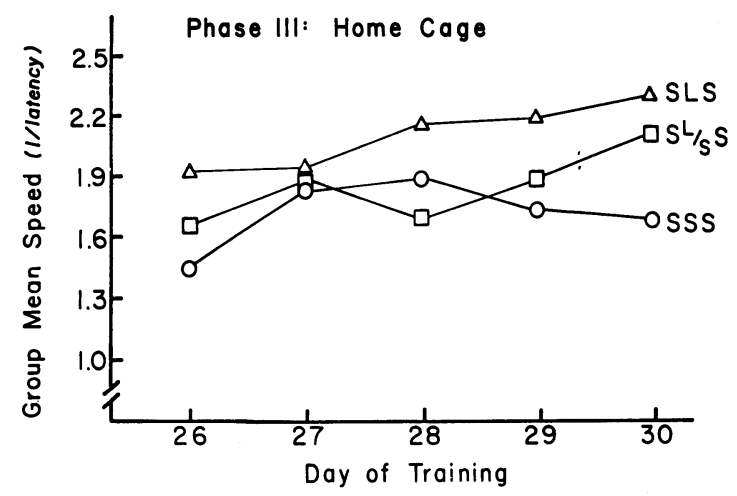

Figure 1. Group mean speeds as a function of days for subjects receiving placement trials in the goalbox. 


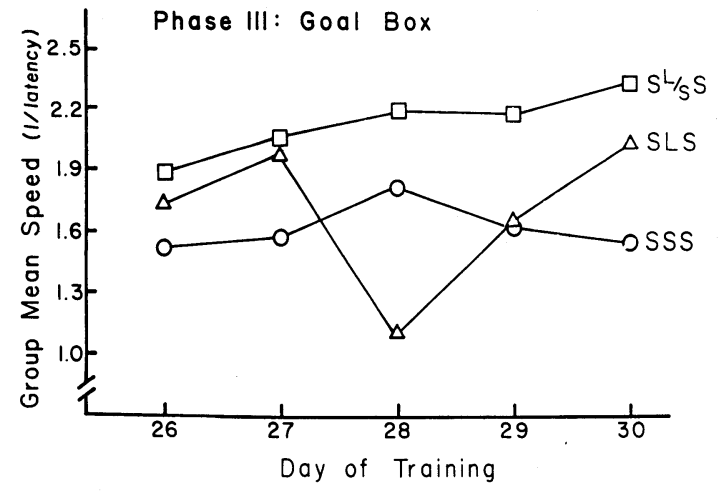

Figure 2. Group mean speeds as a function of days for subjects receiving placement trials in a housing cage.

location $[\mathrm{F}(1,54)=4.24, \mathrm{p}<.05]$, as well as a significant Location by Treatment interaction $[\mathrm{F}(2,54)=7.46$, $\mathrm{p}<.01]$. Tukey (a) paired comparisons between group mean running speeds from Day 28 indicated that Group $\mathrm{SL}_{\mathrm{gb}} \mathrm{S}$ ran significantly slower than its respective control, Group $\mathrm{SS}_{\mathrm{gb}} \mathrm{S}(\mathrm{p}<.05)$. None of the other Tukey (a) paired-comparison tests reached significance (all ps > .05).

\section{DISCUSSION}

The finding of depressed speeds early in the postshift period following a shift to small magnitude of reward is consistent with the results of previous studies (e.g., Capaldi \& Lynch, 1967; Crespi, 1942; Ehrenfreund, 1971; Gonzales et al., 1962). The present study further demonstrated that a NCE or "depression effect" can result even though an instrumental running response to the large magnitude of reward does not occur. However, the present results also showed that a large reward noncontingent on a traversal down the runway produces a depression effect, not during home-cage placements, but, rather, when the reward is received in goalbox placements that have some of the stimulus properties (brightness) of the alley, and solely when the reward is consistent.

The major findings of the present research support a frustration theory account (Amsel, 1958, 1962) of a successive NCE, as opposed to more relativistic or perceptual explanations (i.e., Bevan \& Adamson, 1960; Collier \& Marx, 1959; Helson, 1964), or a generalization-decrement description of the data (Capaldi \& Lynch, 1967).

As discussed earlier, frustration theory states that a group of animals receiving large reward noncontingently during a goalbox placement should experience a build-up in $\mathrm{rg}$-sg or "expectancy" to large reward. The similarity of runway to goalbox stimuli also affects the intensity of the expectancy construct. Therefore, the classically conditioned rg-sg build-up to large reward, acquired during the second phase in the runway goalbox, even in the absence of the running response, should generalize to the runway. In the presence of small reward during the third phase, the conditioned expectancy to large reward (rg-sg) should evoke a frustration response (rf-sf), which temporarily competes with the approach response, thus producing an approach-avoidance conflict that should cause a performance decrement. However, a group of animals that received the large reward in the home cage, thus experiencing the build-up of rg-sg in a setting different from the runway, should not demonstrate a performance decrement when shifted to small reward during the third phase. The marked dissimilarities of home-cage trial settings to the runway should not permit sufficient levels of the conditioned expectancy to large reward ( $\mathrm{rg}$-sg) to generalize to the alley, hence ultimately precluding the NCE from being obtained.

On the other hand, perceptual or adaptation-level theory (Helson, 1964) would postulate a performance decrement or NCE in both of the above groups during the third phase, when shifted to small reward. According to this position, the adaptation level is a function of the animals' total experience with the reward. Thus, whether the animals receive their respective rewards in the runway, goalbox, or home cage is of little consequence. The location of the received reward should not interfere with the development of the adaptation level to large reward during the second phase of the study. When shifted to small reward in the third phase, however, the perception of a reinforcing stimulus (reward) that is smaller than their current adaptation levels for that stimulus should produce the NCE in both groups.

As in previous literature (i.e., Calef, 1972; Calef et al., 1973; Maxwell et al., 1976), Capaldi's (1967) generalization-decrement hypothesis was not supported by the present data. As predicted from this hypothesis, none of the four experimental groups should have shown depressed running speeds in the third phase, due to habit strength having fully accrued to small reward during the first phase. The finding of a significant performance decrement appears to question seriously the validity of the generalization-decrement hypothesis as applied to the NCE in successive contrast paradigms.

It should also be pointed out that frustration theory could possibly account for the absence of the depression effect when varied large and small magnitude of reward were presented during goalbox placement trials. Similar to animals in the Davis and North (1967) study, which displayed an absence of the NCE subsequent to running to varied large and small reward during preshift, animals of the present study, given varied large and small reward during placement trials in the goalbox, may have actually learned to "run" in the presence of frustrative stimuli. However, one would have to assume that some instrumental response associated with or approximating the actual running response may have occurred (such as consummatory responses, extending the paws to grasp the food pellets, or perhaps taking a step in order to adjust to a feeding posture). When shifted to small reward, these animals should continue to run in the presence of frustrative stimuli (rf-sf) and show no NCE.

A more probable explanation for the lack of an observable performance decrement in the third phase of the group of animals receiving varied large and small reward in the goalbox may be found in the phenomenon of "incentive averaging" (McHose, 1970). Generally stated, it is postulated that if varied large and small reward is presented, the animal responds to some average between the two magnitudes, which is assumed to be a less "frustrating" experience for the animal than the postshift decrease from a consistently large reward. For example, a decrease in reward from a consistently large magnitude to a small magnitude (i.e., 12 pellets to 1 pellet) is considered more "frustrating" than a decrease from a varied large and small amount to a small magnitude (i.e., an average of 6.5 pellets shifted to 1 pellet). Therefore, the amount of frustrative stimuli associated with the varied reward magnitude is significantly less than the amount of frustrative stimuli associated with consistently large reward. Accordingly, a performance decrement may not have occurred in the third phase for the varied large and small magnitude of reward group because the shift to small reward was not as "frustrating" as it would be for a group receiving consistently large reward during the analogous phase.

Finally, the results of the present study using a successive shift in magnitude of reward design are not similar to those obtained by Harris, Collerain, Wolf, and Ludvigson (1970), who employed a simultaneous discrimination-contrast paradigm (Bower, 1961). In the Harris et al. (1970) study, subjects receiving large reward magnitude noncontingent on a traversal down the alley, but instead during placements in a setting 
similar to the home cage, showed a S- NCE. This unlikeness in the contrast findings between the two paradigms suggests that the underlying mechanisms producing successive NCEs may not be similar to those producing simultaneous NCEs. Since NCEs were found in "home-cage" placements using a simultaneous paradigm, but not when employing a successiveshift paradigm, this may suggest that perceptual processes underlie the S- NCE, whereas a frustration mechanism is responsible for producing the "depression effect."

\section{REFERENCES}

AMSEL, A. The role of frustrative nonreward in noncontinuous reward. Psychological Bulletin, 1958, 55, 102-119.

AMSEL, A. Frustrative nonreward in partial reinforcement and discrimination learning: Some recent history and theoretical extensions. Psychological Review, 1962, 69, 306-328.

AMSEL, A., \& HANCock, W. Motivational properties of frustration III: Relation of frustration effect to antedating goal factors. Journal of Experimental Psychology, 1957, 53, 126-131.

Bevan, W., \& ADAmson, R. Reinforcers and reinforcement: Their relation to maze performance. Journal of Experimental Psychology, 1960, 59, 226-232.

BLACK, R. W. Shifts in magnitude of reward and contrast effects in instrumental and selective learning: A reinterpretation. Psychological Review, 1968, 75, 114-126.

BOWER, G. H. A contrast effect in differential conditioning. Journal of Experimental Psychology, 1961, 62, 196-199.

CALEF, R. S. The effect of large and small magnitude of intertrial reinforcement on successive contrast effects. Psychonomic Science, 1972, 29, 309-312.

Calef, R. S., Hopkins, D. C., Mchewitt, E. R., \& Maxwell, F. R. Performance to varied reward following continuous reward training in the runway. Bulletin of the Psychonomic Society, 1973, 2, 103-104.

CAPAldi, E. J. A sequential hypothesis of instrumental learning. In K. W. \& J. T. Spence (Eds.), The psychology of learning and motivation (Vol. 1). New York: Academic Press, 1967.
Capaldi, E. J., \& Lynch, D. Repeated shifts in reward magnitude: Evidence in favor of an associational and absolute (non-contextual) interpretation. Journal of Experimental Psychology, 1967, 75, 226-235.

Collier, G., \& MarX, M. H. Changes in performance as a function of shifts in magnitude of reinforcement. Journal of Experimental Psychology, 1959, 57, 305-309.

CRESPI, L. P. Quantitative variations in incentive and performance in the white rat. American Journal of Psychology, 1942, 55, 467-517.

DAvis, S. F., \& NoRTh, A. J. The effect of varied reinforcement training on behavior following incentive reduction. Psychonomic Science, 1967, 9, 395-396.

EHRENFREUND, D. Effect of drive on successive magnitude shift in rats. Journal of Comparative and Physiological Psychology, 1971, 76, 418-423.

Gonzales, R. C., Gleitman, H., \& Bitterman, M. E. Some observations on the depression effect. Journal of Comparative and Physiological Psychology, 1962, 55, 578-581.

Harris, D. R., Collerain, I., Wolf, J. C., \& Ludvigson, H. W. Negative S- contrast with minimally contingent large reward as a function of trial initiation procedure. Psychonomic Science, 1970, 19, 189-190.

Helson, H. Adaptation-level theory: An experimental and systematic approach to behavior. New York: Harper \& Row, 1964.

Ludvigson, H. W., \& GAY, S. Differential reward conditioning: $S$ - contrast as a function of the magnitude of $S 2 / 3$. Psychonomic Science, 1966, 5, 289-290.

Maxwell, F. R., Calef, R. S., Murray, D. W., Shepard, J. C., \& Norville, R. A. Positive and negative successive contrast effects following multiple shifts in reward magnitude under high drive and immediate reinforcement. Animal Learning \& Behavior, 1976, 4, 480-484.

McHose, J. H. Relative reinforcement effects: $S_{1} / S_{2}$ and $S_{1} / S_{1}$ paradigms in instrumental conditioning. Psychological Review, 1970, 77, 135-146.

(Received for publication September 12, 1978.) 